\title{
Comparative mitogenomic analyses of three scallops (Bivalvia: Pectinidae) reveal high level variation of genomic organization and a diversity of transfer RNA gene sets Xiangyun $\mathrm{Wu}$, Xiaodong $\mathrm{Xu}$, Ziniu $\mathrm{Yu}^{*}$ and Xiaoyu Kong
}

\author{
Address: Key Laboratory of Marine Bio-resource Sustainable Utilization, Laboratory of Applied Marine Biology; South China Sea Institute of \\ Oceanology, Chinese Academy of Sciences, 164 West Xingang Road, Guangzhou 510301, PR China \\ Email: XiangyunWu - xywu2007@scsio.ac.cn; Xiaodong Xu - right2468@163.com; Ziniu Yu* - carlzyu@scsio.ac.cn; \\ Xiaoyu Kong - xykong@scsio.ac.cn \\ * Corresponding author
}

Published: 5 May 2009

BMC Research Notes 2009, 2:69 doi:10.1186/1756-0500-2-69

This article is available from: http://www.biomedcentral.com/l756-0500/2/69

(c) 2009 Yu et al; licensee BioMed Central Ltd.

This is an Open Access article distributed under the terms of the Creative Commons Attribution License (http://creativecommons.org/licenses/by/2.0), which permits unrestricted use, distribution, and reproduction in any medium, provided the original work is properly cited.
Received: 26 January 2009

Accepted: 5 May 2009

\begin{abstract}
Background: It can be seen from the available mollusk mitogenomes that the family Pectinidae exhibits the most variation in genome organization. In this study, comparative mitogenomic analyses were performed for three scallops from the subfamily Chlamydinae (Pectinidae), with the goal of characterizing the degree of variability of mitogenome organization and other characteristics among species from the same subfamily and exploring their possible evolution route.

Findings: The complete or nearly complete mtDNA sequences of scallop Mimachlamys nobilis (I7 935 bp), Mizuhopecten yessoensis (20 964 bp) and Chlamys farreri ( 17035 bp) were determined using long PCR amplification and primer walking sequencing strategy. Highly variable size difference of the three genomes resulted primarily from length and number variations of non-coding regions, and the major difference in gene content of the three scallop species are due to varying tRNA gene sets. Only 21 , I6, and I 7 tRNA genes were detected in the mitogenomes of $M$. nobilis, $M$. yessoensis and $C$. farreri, respectively. Remarkably, no trnS gene could be identified in any of the three scallops. A newly-detected trnA-like sequence within the mitogenome of $M$. yessoensis seems to exemplify the functional loss of a tRNA gene, and the duplication of $\operatorname{trn} D$ in $M$. yessoensis raises a fundamental question of whether the retention of the tRNA gene copy of 2-tRNAs is easier than that of 4tRNAs. Analysis of putative evolutionary pathways of gene rearrangement indicates that transposition of neighboring gene blocks may play an important role in the evolution of mitogenomes in scallops. Parsimonious analysis of the genomic variations implies that the mitogenomes of $M$. yessoensis and $C$. farreri are likely to derive independently from a common ancestor that was closely related to $M$. nobilis.
\end{abstract}

Conclusion: Comparative mitogenomic analyses among three species from the subfamily Chlamydinae show that the three genomes exhibit a high level of genomic variation and a diversity of tRNA gene sets, characterized by extensive translocation of genes. These features provide useful clues and information for evolutionary analysis of scallop mitogenomes. 


\section{Findings}

It can be seen from the available mollusk mitogenomes that the Pectinidae exhibits the most variation in genome organization. When we were initiating the current study, three complete or nearly complete mitogenomes, representing three subfamilies, were available from this family, i.e., Argopecten irradians (Aequipectini group, GenBank: EU023915), Mizuhopecten yessoensis (Chlamydinae, GenBank: AB271769) and Placopecten magellanicus (Palliolinae, GenBank: DQ088274). Obvious differences in mitogenome organization of three scallops were observed: 1) the sizes of three mitogenomes are distinct from each other, i.e. 16221 bp for A. irradians, 20414 bp for M. yessoensis and 32115 bp for P. magellanicus [1]; 2) allegedly, the three mitogenomes have significantly different tRNA gene sets, with the numbers of 22, 32 and 9 for A. irradians, P. magellanicus and M. yessoensis, respectively; 3 ) the genomes show distinct gene arrangement patterns, namely unique rearrangements involving nearly every gene.

The degree of gene arrangements of mitogenomes from different species in the same subfamily, Chlamydinae, is one of our concerns. Therefore, in this study the complete mtDNA sequences of Mimachlamys nobilis and M. yessoensis, and nearly complete mtDNA sequence of Chlamys farreri are determined using long PCR amplification and primer walking sequencing strategy (see Additional file 1 ), and used for comparative analyses. Another reason for inclusion of $M$. yessoensis is that its first mitogenome data deposited in GenBank ( $\underline{\mathrm{AB} 271769}$ ) seems to bear significant omissions and mis-annotations of tRNA genes and protein-coding gene. Apparently, these mis-annotations need to be amended for further studies of gene order variation, evolution and phylogenetic analysis.

\section{Genome organization and nucleotide composition}

The size of the mitogenome is $17935 \mathrm{bp}$ for M. nobilis (GenBank: FJ595958). Due to technical difficulties in sequencing, a small part (up to a couple of hundreds base pairs) of the mitogenome of $C$. farreri was not obtained and the nearly complete genome is 17035 bp in length (GenBank: FJ595957). Genome assembly indicated that the unfinished section is the start part of the major noncoding region (MNR). The complete mtDNA sequence of M. yessoensis obtained in this study is 20964 bp in length (GenBank: FJ595959), which is 550 bp longer than the nearly complete genome. Genome assembly indicated that the previously unfinished section is part of the MNR. Annotation to the mitogenome obtained in the current study and a re-annotation to $\underline{\mathrm{AB} 271769}$ revealed the following findings: 1) the "absent" $\operatorname{cox} 2$ gene in previous annotation is actually present, corresponding to nucleotides 14 638-15 325, with "CTG" as initiation codon and " $\mathrm{T}$ " as termination codon; and 2) the genome has a total of 16 tRNA genes, instead of nine identified in that nearly complete genome. Additionally, 56 transitions were detected from a comparison of mitogenomes FJ595959 and AB271769.

The three mitogenomes contain 12 protein-coding genes (PCGs), lacking the atp 8 gene as in most bivalves, two ribosomal RNA genes and varying numbers of tRNA gene set (see Additional file 2; Additional file 3; Additional file 4; Additional file 5; Figure 1). No obvious difference in gene length was observed among three scallops (Additional file 2); thus, what contributes to the differences in

A

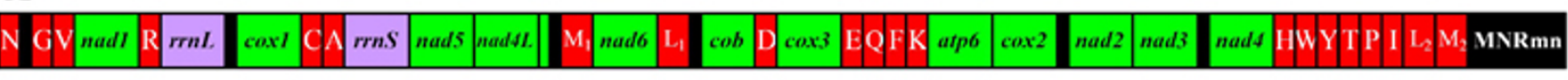

B
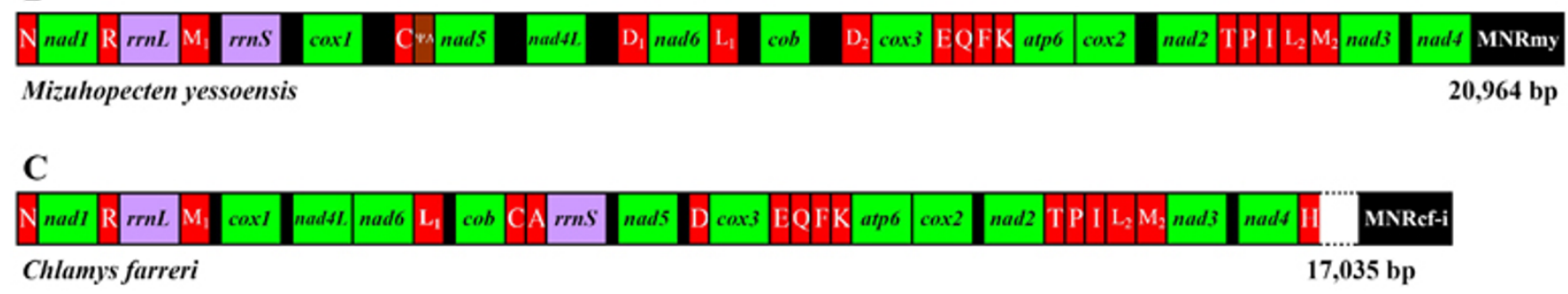

Figure I

Organization of the mitochondrial genome of Mimachlamys nobilis (A), Mizuhopecten yessoensis (B) and

Chlamys farreri (C). Protein and rRNA coding genes are abbreviated as in the text, and transfer RNA genes are depicted by their corresponding one-letter amino acid code. Non-coding regions ( $>50$ bp in length) are labeled by black box and the major non-coding region is designated as "MNRmn", "MNRmy" and "MNRcf-i", respectively. 
genome sizes is primarily the number and length of noncoding regions (NCRs). An interesting finding of this study is the existence of repeat units within the NCR of $M$. yessoensis mtDNA (see Additional file 6). The nucleotide compositions and usage frequencies of entire mitogenome for three scallops have a similar pattern (Additional file 2). The nucleotide compositions are all strongly skewed away from $\mathrm{C}$ in favor of G (the GC-skews are $0.312,0.248$ and 0.319 separately) and from A in favor of $\mathrm{T}$ (the AT-skews are $-0.252,-0.197$ and -0.240 respectively) (Additional file 2). The AT content of the three genomes are $59.6 \%, 55.3 \%$ and $57.9 \%$ in $M$. nobilis, $M$. yessoensis, and C. farreri respectively, lower than the average AT content of 16 available bivalves (61.6\%), but comparable to that of other two pectinids $(57 \%$ for $A$. irradians; $55.7 \%$ for P. magellanicus). Up to date, the five species within the Pectinidae represent the lowest average AT content $(57.1 \%)$ compared to other molluscan lineages (66\% for Gastropoda; 69.4 for Polyplacophora; $71.2 \%$ for Scaphopoda; $71.7 \%$ for Cephalopoda). The AT contents are slightly higher in $r r n L(61.0 \%, 57.8 \%$ and $58 \%$ separately) and lower in $r m S$ (56.7\%, 50.6\% and $52.5 \%)$ than that of each full mitogenome.

\section{Protein-coding genes}

Amino acid identity in proteins for pairs of three scallops ranged from as low as $54.3 \%$ in nad2 between $M$. nobilis and C. farreri to as high as $93.3 \%$ in cox 1 between M. yessoensis and C. farreri (Table 1). M. yessoensis and C. farreri share a higher degree of amino acid similarity in all genes than do between other pairs. According to our data, it appears that the most conserved PCGs are cox1, nad1, and nad3 (identity $>80 \%$ ), and the least conserved is nad2 (identity $=61.1 \%$ ).

Table I: Protein-coding gene assignments and identity of Mimachlamys nobilis (Mnob), Mizuhopecten yessoensis (Myes) and Chlamys farreri (Cfar)

\begin{tabular}{lccc}
\hline Protein & \multicolumn{3}{c}{ \% identity } \\
\cline { 2 - 4 } & Mnob-Myes & Mnob-Cfar & Myes-Cfar \\
\hline atp6 & 65.8 & 65.8 & 75.3 \\
cob & 64.2 & 67 & 80.5 \\
coxl & 87 & 87.4 & 93.3 \\
cox2 & 64.9 & 65.3 & 75.7 \\
cox3 & 72.7 & 74.8 & 86.2 \\
nad1 & 82.2 & 81.2 & 85.4 \\
nad2 & 57.4 & 54.3 & 71.6 \\
nad3 & 75.8 & 77.8 & 89.9 \\
nad4 & 77.2 & 75.8 & 86 \\
nad4L & 72.1 & 71.2 & 85.6 \\
nad5 & 71.9 & 68.6 & 77.3 \\
nad6 & 64.8 & 67.9 & 75.9 \\
\hline
\end{tabular}

In this study, most of PCGs in three mitogenomes use conventional initiation codons (20 for ATG, 10 for ATA and 1 for ATT), but two genes use the alternative ones ( $M$. nobilis: nad1-TTG; $M$. yessoensis: cox2-CTG, nad1-GTG; $C$. farreri: cox2-GTG, nad1-GTG). Notably, one of the alternative initiation codons, GTG was also frequently used in two previously described scallop mitogenomes ( 5 for $A$. irradians and 2 for $P$. magellanicus). Only two genes use CTG as an initiation codon in the 45 reported molluscan mitogenomes (M. yessoensis: cox2; Lottia digitalis: cox 1 ). Interestingly, six of 12 PCGs in M. yessoensis and C. farreri show a derived characteristic in their use of initiation codons when compared with those of $M$. nobilis, i.e. atp6 (ATA $[M$. nobilis $] \rightarrow$ ATG $[M$. yessoensis and C. farreri] $]$ ), cox 1 $(\mathrm{ATA} \rightarrow \mathrm{ATG}), c o b(\mathrm{ATA} \rightarrow \mathrm{ATG})$, nad1 $(\mathrm{TTG} \rightarrow \mathrm{GTG}), n a d 2$ $(\mathrm{ATG} \rightarrow \mathrm{ATA})$ and nad4 (ATG $\rightarrow$ ATA). In general, the usage of initiation codons among three scallops is flexible, but not random.

Three genes stop with identical termination codon in all three scallops, i.e. nad1 (TAG), nad4 (TAG) and nad4L (TAA). Five genes (atp6, cox2, cox3, cob and nad2) share the same termination codon in the mitogenomes of $C$. farreri and $M$. nobili and three genes (cox1, nad 3 and nad5) use the same termination codon in the genomes of M. yessoensis and M. nobilis. The nad6 in M. yessoensis and C. farreri display the derivative feature of termination codon usage when compared with that of M. nobilis (TAA [M. nobilis $] \rightarrow$ TAG $[M$. yessoensis and C. farreri] $]$ ). The cox2 gene in both $M$. yessoensis and $C$. farreri contains a truncated termination codon, ending with a single thymine.

\section{Transfer RNA genes}

Despite an extensive search with the tRNAscan-SE [2] and by eye inspection, only 21,16 , and 17 tRNA genes were detected in the mitogenomes of $M$. nobilis, M. yessoensis and C. farreri, respectively (Additional file 7; Additional file 8; Additional file 9). Particularly, no trnS gene could be identified in any of the three scallops, and four tRNAs (trnG, $\operatorname{trn} V$, trn $W$ and $\operatorname{trn} Y$ ) were absent in the mitogenome of both $M$. yessoensis and C. farreri. Nevertheless, we presume that the loss of trnW and trnY in the mitogenome of $C$. farreri may be an artifact, due to unfinished sequencing of the region downstream of $t r n H$. The loss of trnS may present the ancestral state for all three scallops of the subfamily Chlamydinae. The loss of the tRNA cluster "GV" in the mitogenome of M. yessoensis and C. farreri may present the first step of tRNA gene loss from the common ancestor of these two species belonging to the same tribe (Chlamydini). Additional derived features of tRNA gene loss and translocations were observed separately for both $M$. yessoensis and C. farreri, e.g. the deletions of $\operatorname{trn} H, \operatorname{trn} W, \operatorname{trn} Y$ and $\operatorname{trn} A$, and the duplication of $\operatorname{trn} D$ in the mitogenome of M. yessoensis. 
Another interesting finding of this study is the identification of a trnA-like sequence within the mitogenome of $M$. yessoensis. For confirmation of this finding, at least three individuals of M. yessoensis were used separately for amplification and sequencing of this fragment. This $\operatorname{trn} A$-like structure is just located following the $\operatorname{trn} C$, where the tRNA gene cluster "CA" has been observed in the mitogenome of both $M$. nobilis and $C$. farreri. Alignment of this trnA-like sequence of $M$. yessoensis with trnA gene sequences of other four available scallops (A. irradians, $P$. magellanicus, $M$. nobilis and $C$. farreri) shows that it shares similar nucleotide sequences in amino acid arm, DHU arm, and anticodon arm with those of $\operatorname{trn} A$ genes, with a distinct sequence in the anticodon loop (Figure 2A). We therefore define this trnA-like genomic region as a pseudotRNA gene (PsA). The existence of PsA may provide evidence of intermediate status of tRNA gene loss. Several mechanisms have been proposed for tRNA gene loss [35]. In this case, trnA gene loss seems fit the model that tRNA genes are gradually lost functionally, and then physically, over long evolutionary periods of time. Gene functional loss via mutation of the anticodon loop may play the most important role in this case.

In the mitogenomes of metazoan, almost all amino acids codons but leucine and serine are decoded by only one tRNA each [6]. The presence of two trnM genes was reported in the mitogenomes of tunicates [7]. However, it is a common phenomenon that mitogenomes of most bivalves contain two trnM genes. In this study, the $\operatorname{trn}_{2}$ gene was found clustered with other tRNA genes, but the locations of $t r n M_{1}$ were variable over species. Another case of tRNA gene duplication is the trnD in M. yessoensis, which is found in single copy in the mitogenomes of both M. nobilis and C. farreri. Both $\operatorname{trn} D_{1}$ and $t r n D_{2}$ are considered true tRNA genes, based on sequence comparison (Figure 2B) as well as their putative secondary structures (Additional file 8). In order to probe into the mechanism of the retention of two copies of non-trnL/trnS tRNA genes, we examined the mitogenomes of all available mollusks for their tRNA gene usage, and found that a total of eight tRNA genes (trnD, trnE, trnF, trnK, trnM, trnN, $\operatorname{trn} Q, \operatorname{trn} V$ ) appear in at least two copies for each (see Additional file 10). Notably, all but trnV have anticodons corresponding to the 2 -fold degenerate codons. This finding raises a fundamental question of whether the retention of the tRNA gene copy of 2-tRNAs is easier than that of 4-tRNAs (also see [6]). Perhaps there are differences in the gene loss and import mechanisms from the cytoplasm to the mitochondria of 4-tRNAs and 2-tRNAs. It is desirable to investigate the mechanisms of tRNA gene loss in further studies; more mitogenomes with variable tRNA gene sets should be included in future studies to draw a solid conclusion.

\section{Putative evolutionary pathway of gene rearrangements}

It is often difficult to trace the evolutionary pathway of metazoan mitogenomes due either to the generally low number of reorganization events (e.g., mammals) or drastic reorganizations in some animal lineages (e.g., nema-
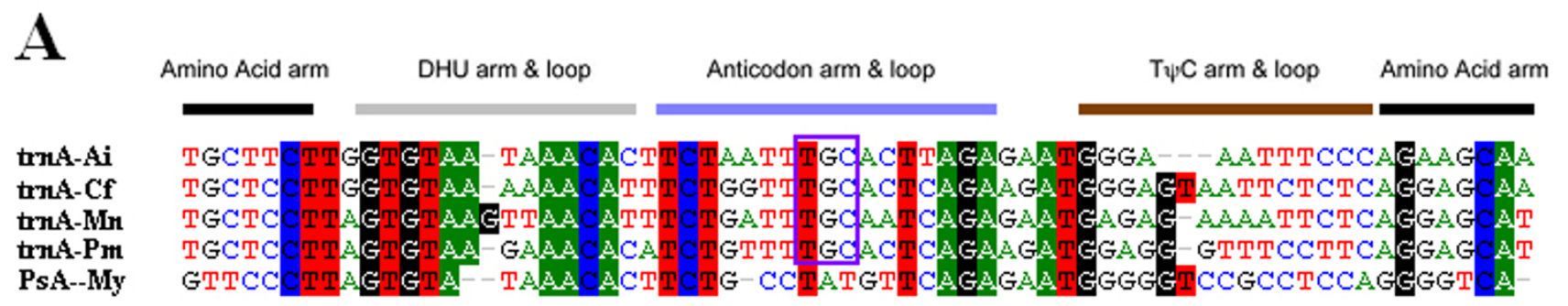

B

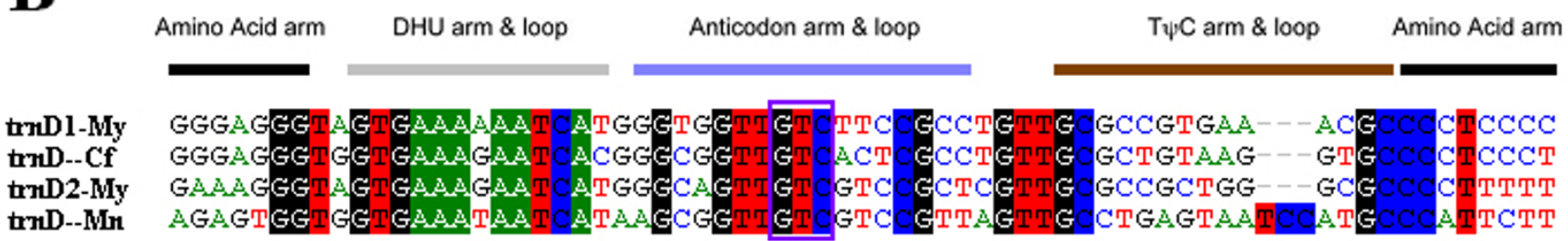

\section{Figure 2}

Alignment of the trnA and pseudogene (PsA) in mitochondrial genomes of five scallops (A), and alignment of trnD in mitochondrial genomes of the three scallops (B). tRNA secondary structure is designed above the alignment, and the position of the anticodon is highlighted with rectangular frame. Ai: Argopecten irradians Cf: Chlamys farreri Mn: Mimachlamys nobilis My: Mizuhopecten yessoensis Pm: Placopecten magellanicus. 
todes, snails and brachiopods etc; see $[8,9])$, particularly in bivalves [e.g., [10,11]]. Phylogentic analyses for five scallops, using the concatenated amino acid sequences of 12 PCGs, resulted a high supported relationship which can be depicted as (A. irradians, (P. magellanicus, (M. nobilis, (M. yessoensis, C. farreri)))) (see Additional file 11). Based on the inferred phylogeny, a putative evolutionary pathway of gene rearrangement in the three species is assumed, and our preferred scenario of mitogenome evolution is further elaborated in Figure 3. In detail, there are at least three permutations between the mitogenomes of $M$. nobilis and that of a putative common ancestor of $M$. yessoensis and C. farreri: transposition of $\operatorname{trn} \mathrm{M}_{1}$; transposition of the tRNA gene cluster "TPIL ${ }_{2} \mathrm{M}_{2}$ ", and loss of trnG and $t r n V$. The tRNA gene cluster "HWY" is assumed to have been present in the putative common ancestor, based on the fact that trnH is just downstream of nad4 in C. farreri. Additionally, the absence of trnW and trnY may be artificial due to the incompletely sequenced downstream region. At least four independent events occurred in the mt gene rearrangement of $M$. yessoensis in the proc- ess of derivation from the putative common ancestor: deletion of the tRNA gene cluster "HWY", transposition of gene block "cox1-trnC-trnA" with rrnS, duplication of trnD and the generation of pseudogene PsA. Only one step is then necessary for the rearrangement of $C$. farreri from the common ancestor, i.e. the transposition of the large fragment "trnC-trnA-rrnS-nad5" with its neighbor block "nad4L-nad6-trnL $L_{1}$-cob".

It has been commonly recognized that variations in gene order are relatively rare in mitogenomes of most metazoan lineages [8]. Dowton et al. [12] estimated a probability of $1 / 2664$ for a single event of gene translocation occurring independently in two mitogenomes (starting from the same gene order in both). However, this probability could be an underestimate according to yet unidentified constraints on modes of gene rearrangements, and it should be applied cautiously. Pectinid bivalves seem to represent another example, as five sequenced mitogenomes exhibit significant genomic rearrangements, suggesting that gene rearrangements occurred frequently

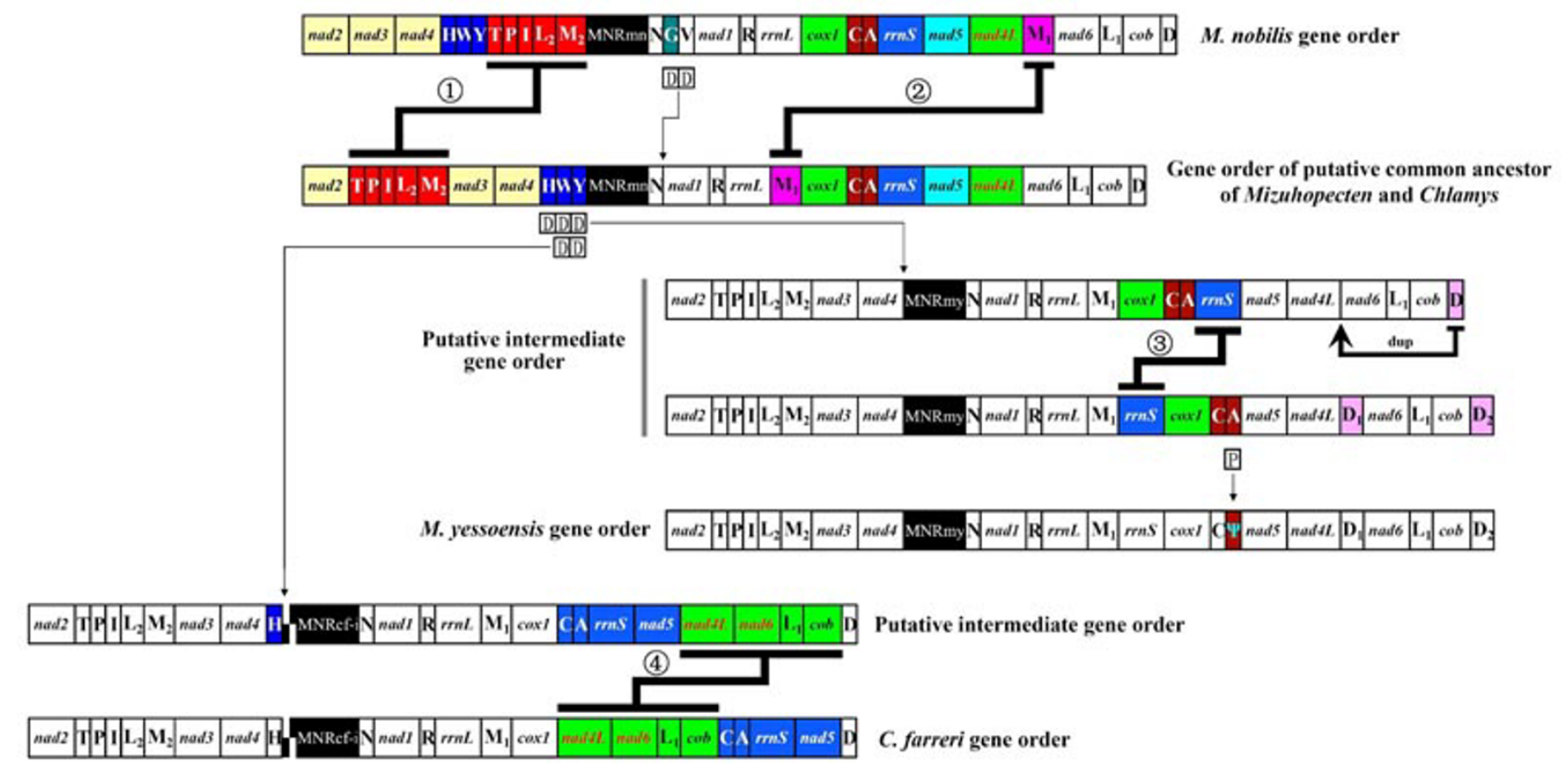

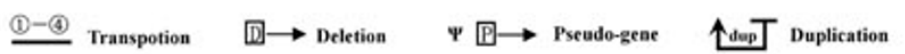

Figure 3

The putative evolutionary pathway of mitochondrial genomes for three scallops. The hypothetical pathway with the minimum number of gene duplication, deletion and transposition is illustrated. A putative common ancestor of $M$. yessoensis and $C$. farreri is shown as it can be most easily and parsimoniously explained. Putative intermediate gene order between "common ancestral gene order" and "M. yessoensis/C. farreri gene order" is also shown to explain the most parsimoniously possible evolutionary pathway of these two species. Symbols are explained under the figure and gene abbreviations are designated as in the text. Genes with identical order in the lineage are put in black/white boxes. 
among lineages in this family. On the other hand, high amino acid sequence identity between $M$. yessoensis and $C$. farreri indicate that these species may have diverged only recently. As illustrated in Figure 3, transposition of neighbor gene blocks (transposition 1, 3 and 4) may play an important role in the evolution of scallop mitogenomes. There is no doubt that $\mathrm{mt}$ genomic rearrangements are in most cases appropriate markers to resolve both ancient and recent divergence processes [6], but the result of this study implies that a careful estimation of the rearrangement pathway is especially required in analyzing the highly variable organizations of mitogenomes in the Pectinidae. However, the scallop mitogenomes with such diversity in their organization seem to be a good model to elucidate molecular evolutionary and phylogenetic issues of mitogenomes in future studies.

\section{Competing interests}

The authors declare that they have no competing interests.

\section{Authors' contributions}

XW designed the research, carried out most of the experiments, performed the data analyses and drafted the manuscript; XX and XK carried out part of the experiments and participated in data analyses; ZY initiated, led the research, supervised all laboratory work and finalized the manuscript. All authors have read and approved the final manuscript.

\section{Additional material}

\section{Additional file 1}

Methods of PCR, sequencing and sequence analyses. Detailed methods for molecular experiments and data analyses are described in this file. Click here for file

[http://www.biomedcentral.com/content/supplementary/17560500-2-69-S1.doc]

\section{Additional file 2}

Basic information of mitochondrial genomes of three scallops. This table presents the positions and nucleotide sequence lengths of mitochondrial genomes of three scallops, and initiation and termination codons for protein-coding genes as well as tRNA gene anticodons (starting from $\operatorname{trnN}$ ).

Click here for file

[http://www.biomedcentral.com/content/supplementary/17560500-2-69-S2.doc]

\section{Additional file 3}

Organization of the mitochondrial genome of Mimachlamys nobilis. Protein and $r R N A$ coding genes are abbreviated as in the text, and transfer RNA genes are depicted by their corresponding one-letter amino acid code. Non-coding regions ( $>50 \mathrm{bp}$ in length) are labeled and the major non-coding region is designated as "MNRmn".

Click here for file

[http://www.biomedcentral.com/content/supplementary/17560500-2-69-S3.jpeg]

\section{Additional file 4}

Organization of the mitochondrial genome of Mizuhopecten yessoensis. Protein and rRNA coding genes are abbreviated as in the text, and transfer RNA genes are depicted by their corresponding one-letter amino acid code. Non-coding regions ( $>50 \mathrm{bp}$ in length) are labeled and the major non-coding region is designated as "MNRmy".

Click here for file

[http://www.biomedcentral.com/content/supplementary/17560500-2-69-S4.jpeg]

\section{Additional file 5}

Organization of the mitochondrial genome of Chlamys farreri. Protein and rRNA coding genes are abbreviated as in the text, and transfer RNA genes are depicted by their corresponding one-letter amino acid code. Non-coding regions ( $>50 \mathrm{bp}$ in length) are labeled and the incomplete sequenced major non-coding region is designated as "MNRcf- $i$ ".

Click here for file

[http://www.biomedcentral.com/content/supplementary/17560500-2-69-S5.jpeg]

\section{Additional file 6}

Analyses of non-coding regions and repeat units in mitogenomes of three scallops. This section describes the unique character of non-coding regions and repeat units in mitogenomes of three scallops, including figure and references.

Click here for file

[http://www.biomedcentral.com/content/supplementary/17560500-2-69-S6.doc]

\section{Additional file 7}

Putative secondary structures for the 21 transfer RNA genes of the Mimachlamys nobilis mitogenome. This figure show the putative secondary structures of tRNA generated by tRNAscan-SE 1.21.

Click here for file

[http://www.biomedcentral.com/content/supplementary/17560500-2-69-S7.jpeg]

\section{Additional file 8}

Putative secondary structures for the 16 transfer RNA genes of the Mizuhopecten yessoensis mitogenome. This figure show the putative secondary structures of tRNA generated by tRNAscan-SE 1.21.

Click here for file

[http://www.biomedcentral.com/content/supplementary/17560500-2-69-S8.jpeg]

\section{Additional file 9}

Putative secondary structures for the 17 transfer RNA genes of the Chlamys farreri mitogenome. This figure show the putative secondary structures of tRNA generated by tRNAscan-SE 1.21.

Click here for file

[http://www.biomedcentral.com/content/supplementary/17560500-2-69-S9.jpeg]

\section{Additional file 10}

List of tRNA usage frequency in 45 available mollusk mitogenomes. This table shows the tRNA usage frequency in 45 available mollusk mitogenomes.

Click here for file

[http://www.biomedcentral.com/content/supplementary/17560500-2-69-S10.pdf] 


\section{Additional file 11}

Phylogentic analyses for five scallops, using the concatenated amino acid sequences of 12 PCGs. Phylogenetic inferring were carried out using MEGA 4.1 for NJ (neighbor-joining), PAUP* $4 b 10$ for MP (maximum parsimony) and PhyML for ML (maximum likelihood).

Click here for file

[http://www.biomedcentral.com/content/supplementary/17560500-2-69-S11.jpeg]

\section{Acknowledgements}

This work was financially supported by the CAS/SAFEA International Partnership Program for Creative Research Teams, Guangdong Science Foundation (No. 845 I03010 I00 I 660) and National Science Foundation of China (No. 40776088).

\section{References}

I. Smith DR, Snyder M: Complete mitochondrial DNA sequence of the scallop Placopecten magellanicus: Evidence of transposition leading to an uncharacteristically large mitochondrial genome. J Mol Evol 2007, 65:380-39l.

2. Lowe TM, Eddy SR: A program for improved detection of transfer RNA genes in genomic sequence. Nucl Acids Res 1997, 25:955-964.

3. Adams KL, Palmer JD: Evolution of mitochondrial gene content: gene loss and transfer to the nucleus. Mol Phylogenet Evol 2003, 29:380-395.

4. Borner GV, Morl M, Janke A, Paabo S: RNA editing changes the identity of a mitochondrial tRNA in marsupials. EMBO J 1996, I 5:5949-5957.

5. Small I, Akashi K, Chapron A, Dietrich A, Duchene AM, Lancelin D, Marechal-Drouard L, Menand B, Mireau H, Moudden Y, Ovesna J, Peeters N, Sakamoto W, Souciet G, Wintz H: The strange evolutionary history of plant mitochondrial tRNAs and their aminoacyl-tRNA synthetases. J Hered 1999, 90:333-337.

6. Podsiadlowski L, Braband A, Mayer G: The Complete mitochondrial genome of the onychophoran Epiperipatus biolleyi reveals a unique transfer RNA set and provides further support for the ecdysozoa hypothesis. Mol Biol Evol 2008, 25:42-5I.

7. Gissi C, lannelli F, Pesole G: Complete mtDNA of Ciona intestinalis reveals extensive gene rearrangement and the presence of an atp8 and an extra trnM gene in ascidians. J Mol Evol 2004, 58:376-389.

8. Boore JL: Animal mitochondrial genomes. Nucl Acids Res 1999, 27:1767-1780.

9. Noguchi $Y$, Endo K, Tajima F, Ueshima R: The mitochondrial genome of the brachiopod Laqueus rubellus. Genetics 2000, I 55:245-259.

10. Serb JM, Lydeard C: Complete mtDNA sequence of the North American freshwater mussel, Lampsilis ornata (Unionidae): An examination of the evolution and phylogenetic utility of mitochondrial genome organization in Bivalvia (Mollusca). Mol Biol Evol 2003, 20: I854-1866.

II. Boore JL, Medina M, Rosenberg LA: Complete sequences of the highly rearranged molluscan mitochondrial genomes of the scaphopod Graptacme eborea and the bivalve Mytilus edulis. Mol Biol Evol 2004, 21 : I 492-1503.

12. Dowton M, Castro LR, Austin AD: Mitochondrial gene rearrangements as phylogenetic characters in the invertebrates: The examination of genome "morphology". Invertebr Syst 2002, I 6:345-356.

\begin{tabular}{|} 
Publish with BioMed Central and every \\
scientist can read your work free of charge \\
"BioMed Central will be the most significant development for \\
disseminating the results of biomedical research in our lifetime. " \\
Sir Paul Nurse, Cancer Research UK \\
Your research papers will be: \\
• available free of charge to the entire biomedical community \\
• peer reviewed and published immediately upon acceptance \\
• cited in PubMed and archived on PubMed Central \\
• yours - you keep the copyright \\
Submit your manuscript here: \\
http://www.biomedcentral.com/info/publishing_adv.asp
\end{tabular}

